\title{
Long-term Photometric Monitoring of FUor and FUor-like Objects
}

\author{
E. Semkov ${ }^{1 *}$, S. Ibryamov ${ }^{2}$, S. Peneva ${ }^{1}$, A. Mutafov ${ }^{1}$ \\ ${ }^{1}$ Institute of Astronomy and National Astronomical Observatory, Bulgarian Academy of \\ Sciences, Sofia, Bulgaria, \\ ${ }^{2}$ Department of Theoretical and Applied Physics, Faculty of Natural Sciences, University of \\ Shumen, Shumen, Bulgaria \\ ${ }^{*} E-$ mail : esemkov@astro.bas.bg
}

\begin{abstract}
A phenomenon with a significant role in stellar evolution is the $\mathrm{FU}$ Orionis (FUor) type of outburst. The first three (classical) FUors (FU Ori, V1515 Cyg and V1057 Cyg) are well-studied and their light curves are published in the literature. But recently, over a dozen new objects of this type were discovered, whose photometric history we do not know well. Using recent data from photometric monitoring and data from the photographic plate archives we aim to study, the long-term photometric behavior of FUor and FUor-like objects. The construction of the historical light curves of FUors could be very important for determining the beginning of the outburst, the time to reach the maximum light, the rate of increase and decrease in brightness, the pre-outburst variability of the star. So far we have published our results for the light curves of V2493 Cyg, V582 Aur, Parsamian 21 and V1647 Ori. In this paper we present new data that describe more accurate the photometric behavior of these objects. In comparing our results with light curves of the well-studied FUors (FU Ori, V1515 Cyg and V1057 $\mathrm{Cyg}$ ), we conclude that every object shows different photometric behavior. Each known FUor has a different rate of increase and decrease in brightness and a different light curve shape.
\end{abstract}

Keywords: stars: pre-main sequence - stars: variables: T Tauri, Herbig Ae/Be - stars: individual: V2493 Cyg, V582 Aur, V1647 Ori, Parsamian 21

\section{Introduction}

During the pre-main sequence (PMS) stage of evolution, young stellar objects exhibit different types of photometric variability (Herbst et al. 1994). One of the most dramatic of these events, with very high amplitude 
variations, is the FU Orionis outburst. First Ambartsumyan (1971) draws attention to this object by linking it to the evolution of the PMS stars and proposes the abbreviation FUor. Herbig (1977) defined FUors as a class of young stellar objects after the discovery of two new FUor stars: V1057 Cyg and V1515 Cyg. The main characteristics of FUors are a significant increase in optical brightness of about 4-5 magnitudes, a F-G supergiant spectrum with broad blue-shifted Balmer lines, strong infrared excess, location in starforming regions, and connection with reflection nebulae (Reipurth \& Aspin 2010, Audard et al. 2014, Connelley \& Reipurth 2018). Typical spectroscopic properties of FUor objects include a gradual change in the spectrum from earlier to later spectral type from the blue to the infrared, a strong $\mathrm{Li}$ I 6707 line, $\mathrm{P}$ Cygni profiles of $\mathrm{H} \alpha$ and $\mathrm{Na}$ I 5890/5896 lines, and the presence of CO bands in the near infrared spectra (Herbig 1977, Connelley \& Reipurth 2018). The prototypes of FUors seem to be low-mass PMS objects ( $\mathrm{T}$ Tauri stars) with massive circumstellar disks.

According to a commonly accepted view, the FUor outburst is produced by a sizable increase in accretion from the circumstellar disk to the stellar surface (Hartmann \& Kenyon 1996). The cause of this increase in accretion from $\sim 10^{-7} M_{\odot} /$ yr to $\sim 10^{-4} M_{\odot} /$ yr appears to be thermal or gravitational instability in the circumstellar disk. This accretion disk model can account for most of the main properties of FUors.

Among all objects associated with the group of FUors, only three (FU Ori, V1057 Cyg, and V1515 Cyg) have detailed photometric observations taken during the outburst and during the fading period (Clarke et al. 2005). For a few objects, V1735 Cyg (Elias 1978, Peneva et al. 2009), V733 Cep (Reipurth et al. 2007, Peneva et al. 2010), V2493 Cyg (Semkov et al. 2010, 2012), V582 Aur (Semkov et al. 2013) the presence of an optical outburst is also documented and they are labeled classical FUors. About a dozen objects have spectroscopic properties similar to the classical FUors, but there is no evidence of an outburst at optical wavelengths. These objects are termed FUor-like (Reipurth et al. 2002, Greene et al. 2008) and only partial photometric observations have been published for them.

\section{Observations}

The photometric observations of FUor and FUor-like objects were performed with the $2 \mathrm{~m}$ RCC, the $50 / 70 \mathrm{~cm}$ Schmidt and the $60-\mathrm{cm}$ Cassegrain telescopes of the National Astronomical Observatory Rozhen (Bulgaria) and with the $1.3 \mathrm{~m} \mathrm{RC}$ telescope of the Skinakas Observatory ${ }^{1}$ of the Institute of Astronomy, University of Crete (Greece). The observations were per-

\footnotetext{
${ }^{1}$ Skinakas Observatory is a collaborative project of the University of Crete, the Foundation for Research and Technology - Hellas, and the Max-Planck-Institut für Extraterrestrische Physik.
} 
formed with eight different types of CCD cameras Photometrics AT200 and VersArray 1300B at the 2-m RCC telescope; Photometrics CH360 and ANDOR DZ436-BV at the 1.3-m RC telescope; SBIG ST-8, SBIG STL-11000M and FLI PL16803 at the 50/70-cm Schmidt telescope; FLI PL09000 at the 60-cm Cassegrain telescope. The technical parameters and specifications of the used CCD are summarized in Ibryamov et al. (2015). All frames were exposed through a set of standard Johnson-Cousins filters. All the data for each object were analyzed using the same apertures and the same background annulus. In this way, we have reached the maximum correspondence of data obtained from different telescopes.

In order to facilitate transformation from instrumental measurement to the standard Johnson-Cousins system for each of the studied objects we are calibrated a sequence of photometric standards. Calibration in BVRI bands was made during several completely clear nights with the $1.3-\mathrm{m} \mathrm{RC}$ telescope in Crete, where the conditions for precise photometric observations are very good.

\section{Archival photographic observations}

The construction of the historical light curves of FUors could be very important for determining the exact moment of the beginning of the outburst and the time to reach the maximum light. Another important option is to study the pre-outburst variability of the star. The only possibility for such a study is a search in the photographic plate archives at the astronomical observatories around the world. Most suitable for this type of research are the plate archives of the big Schmidt telescopes that have a large field of view. Photographic observations from BAO could also be used for these purposes after the implementation of the Byurakan Astrophysical Observatory Plate Archive Project (Mickaelian et al. 2017).

In this paper, we present some of our result using the photographic plate archives of the 105/150 cm Schmidt telescope at Kiso Observatory (Japan), the $67 / 92 \mathrm{~cm}$ Schmidt telescope at Asiago Observatory (Italy) and the 2-m RCC and the 50/70 cm Schmidt telescopes at NAO Rozhen (Bulgaria). We also used the digitized plates from the Palomar Schmidt telescope, available via the website of the Space Telescope Science Institute.

The plates from Asiago Schmidt telescope are inspected visually through a high-quality Carl Zeiss microscope offering a variety of magnifications. The magnitude is then derived by comparing the stars in the photometric sequence with the variable, identifying those that are more closely bracketing the variable. The plates from Kiso Schmidt telescope were scanned with Canon CanoScan LiDE 600F portable scanner, which has 1200 dpi resolution. The plates from NAO Rozhen were scanned with Epson Expression 1640 XL/10000XL scanners, which have 1600-2400 dpi resolution and a cor- 
responding pixel size from $16 \times 16 \mu \mathrm{m}$ to $10 \times 10 \mu \mathrm{m}$. Aperture photometry of the digitized plates was performed with DAOPHOT routines using the same aperture radius and the background annulus as for CCD photometry.

\section{Results and discussion}

\subsection{V2493 Cyg}

The large amplitude outburst of V2493 Cyg was discovered during the summer of 2010 (Semkov \& Peneva 2010, Miller et al. 2011, Semkov et al. 2012). The new FUor candidate is located in the dark clouds (so-called "Gulf of Mexico") between NGC 7000 and IC 5070 a region of active star formation. Simultaneously with the optical outburst appearance of a reflection nebula around V2493 Cyg typical of all classical FUor objects was observed. Follow-up photometric observations recorded an ongoing light increase in both the optical and infrared (Kóspál et al. 2016, Semkov et al. 2017). The high and low resolution spectroscopic observations by Miller et al. (2011), Lee et al. (2015), Semkov et al. (2017) showed significant variability in both the profiles and intensity of the spectral lines. On the basis of our photometric monitoring and the spectral properties at the maximal light (a G3I supergiant spectrum with strong P Cyg profiles of $\mathrm{H} \alpha$ and $\mathrm{Na}$ I D lines), we have confirmed that the observed outburst of V2493 Cyg is of FUor type (Semkov et al. 2012).

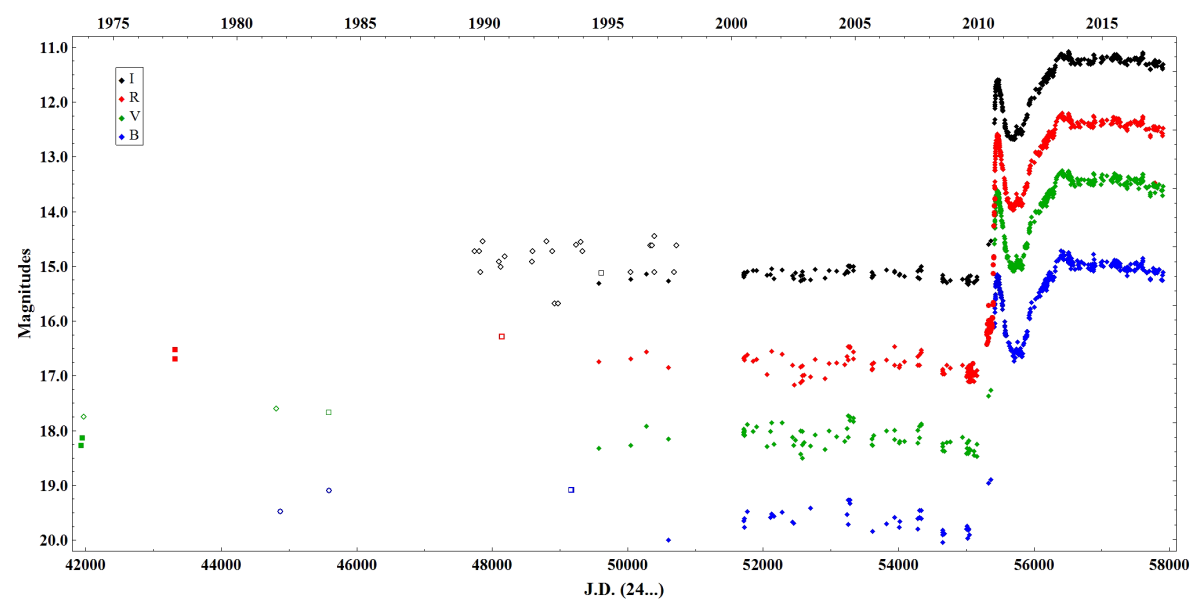

Figure 1: Long term $B V R I$ light curves of V2493 Cyg.

The BVRI light-curves of V2493 Cyg during the period September 1973 - May 2017 are plotted in Fig. 1. The filled diamonds represent our CCD observations, the filled circles CCD observations from the Palomar Schmidt telescope, the open diamonds photographic data from the Asiago Schmidt telescopes, the open squares photographic data from the Palomar Schmidt 
telescope, the filled squares photographic data from the Byurakan Schmidt telescope and the open circles photographic data from the Rozhen 2-m RCC telescope. The photometric observation obtained before the outburst displayed only small amplitude variations in all pass-bands typical of $\mathrm{T}$ Tauri stars. The observational data indicate that the outburst started sometime before May 2010, and reached the first maximum in September/October 2010. Since October 2010, a slow fading was observed and up to May 2011 the star brightness decreased by 1.4 mag. $(V)$. Since the autumn of 2011 , another light increase occurred and the star became brighter by 1.8 mag. $(V)$ until April 2013. During the period April 2013 - May 2017 the star continues to keep its maximum brightness showing a little bit fluctuations around it. V2493 Cyg was the first FUor object observed in all spectral ranges during the rise of the brightness as well as during the first year after reaching the maximum brightness.

\subsection{V582 Aur}

An object with a very strong increase in brightness is discovered by Anton Khruslov (Samus 2009) in the region of active star formation near Auriga OB2 association. The star was cataloged as USNO A2.0 1200-03303169 and according to the General Catalog of Variable Stars as V582 Aur. Munari et al. (2009) obtained the first low-resolution spectrum of V582 Aur and registered the presence of absorption lines of the Balmer series, Na I D and $\mathrm{Ba}$ II ( $\lambda$ 6496) and the absence of the Li I ( $\lambda$ 6707) line in the spectrum. Our results from detailed photometric and spectral observations of the star were reported in Semkov et al. (2013). In the paper we come to the conclusion that V582 Aur has all observational characteristics of FUor objects.

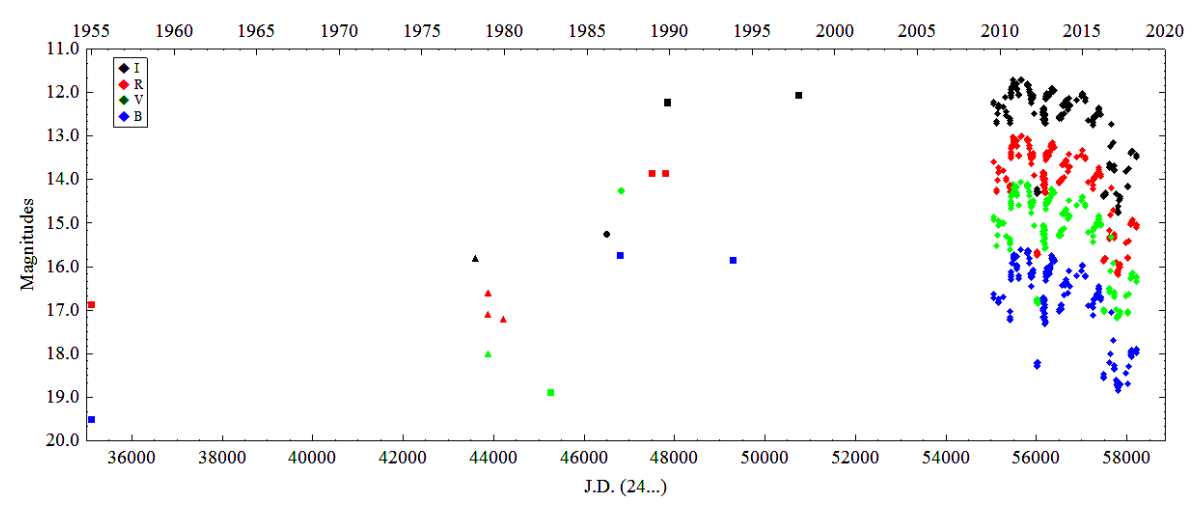

Figure 2: Long term $B V R I$ light curves of V582 Aur.

The historical $B V R I$ light curves of V582 Aur from all available photometric observations are plotted in Fig. 2. On the figure, the filled diamonds represent our $\mathrm{CCD}$ observations, the filled circles photographic data from 
the $67 / 92 \mathrm{~cm}$ Asiago Schmidt telescope, the filled triangles photographic data from the 105/150 cm Kiso Schmidt telescope, and the filled squares photographic data from the Oschin Schmidt Telescope on Palomar. Typical for this FUor object is a very strong variability with a large amplitudes. During the large drop in brightness on 2012, the spectrum of V582 Aur change from a typical FUor to typical $\mathrm{T}$ Tauri star spectrum. And also during this period the star indicate the typical for UX Orionis objects effect of color reversal due to the appearance of dust particles in the circumstellar environment (Semkov et al. 2013).

\section{$4.3 \quad$ V1547 Ori}

The outburst of PMS star V1647 Ori was discovered by amateur astronomer Jay McNeil in January 2004 (McNeil 2004). The star showed an increase in optical brightness by around 5 mag. beginning from November 2003 till February-March 2004 (Briceño et al. 2004). V1647 Ori remains in a state of maximal light about two years, then its brightness declined to the pre-outburst level. During the outburst V1647 Ori exhibited a strong emission spectrum in the optical and near-IR, typical for eruptive PMS stars from EXor type (Herbig 2008). A second outburst of the star was registered in 2008, when its brightness increased again to the level of the first eruption (Kun 2008). The optical and infrared follow-up observations show that the star and the surrounding nebula appear photometrically and morphologically similar to the first outburst.

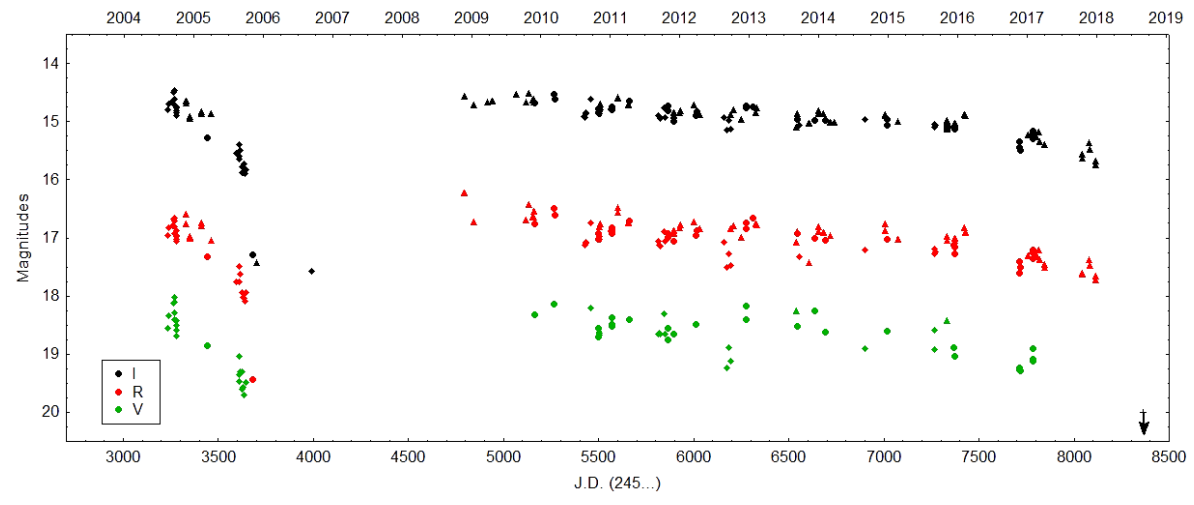

Figure 3: VRI light curves of V1547 Ori during the first and second outburst. Only data from our photometric monitor are plotted. The observation limit of the 2-m RCC telescope (September 1 and 2, 2018) is indicated by an arrow.

Our observations suggest that the second outburst of V1647 Ori continued for approximately ten years. At the beginning of September 2018, we recorded a new drop in the brightness of the object, which is below the 
2-m RCC telescope I-band limit. The photometric data show a continuous slight decrease in brightness during the period from the end of 2008 to the beginning of 2018. In the meantime the star becomes redder with decreasing of brightness. According to Aspin et al. (2009) the decrease in brightness of V1647 Ori over the period 2006-2008 was caused by reduction in accretion rate and reformation of dust in the circumstellar environment of the star. Such interpretation of the observational results leads Aspin et al. (2009) to the hypothesis that V1647 Ori is a FUor object.

\subsection{Parsamian 21}

Parsamian 21 is a young stellar object surrounded by an extended reflection nebula, located in a small dark cloud in Aquila. The object was discovered on the plates from the Palomar Observatory Sky Survey and included in the catalog of cometary nebulae (Parsamian 1965). On the basis of optical spectroscopic and far-infrared properties Staude \& Neckel (1992) suggest affiliation of Parsamian 21 to the group of FUor objects. Results supporting the FUor nature of Parsamian 21 were published in Kóspál et al. (2008). Parsamian 21 was a subject of many studies, but very few optical photometric data have been published to the present. Since no outburst was observed at optical wavelengths in most of the studies Parsamian 21 is classified as FUor-like object (Greene et al., 2008).

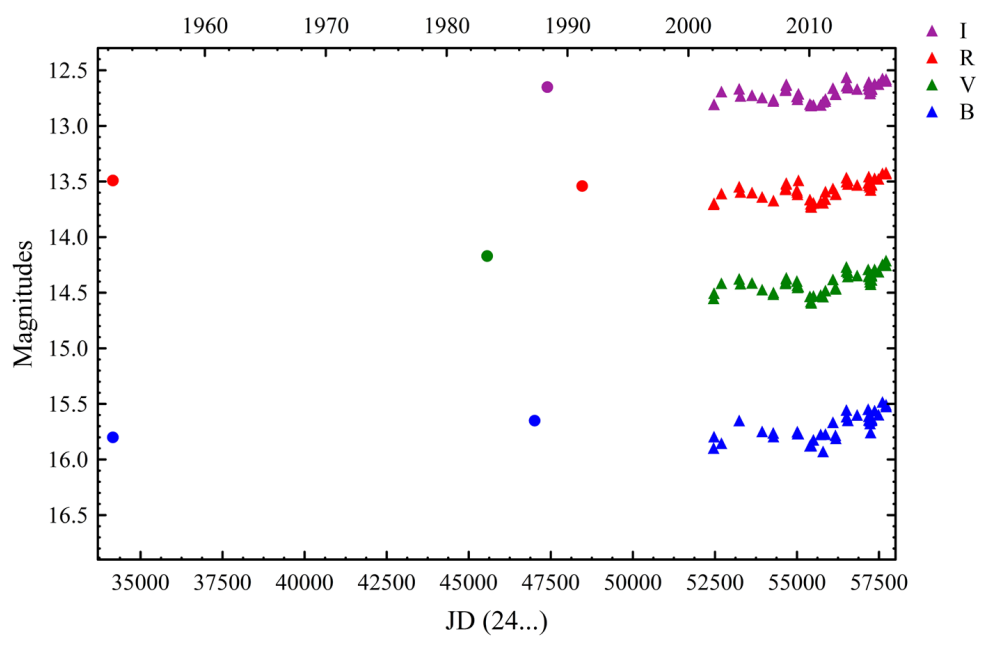

Figure 4: Long term $B V R I$ light curves of Parsamian 21. The filled triangles represent our CCD observations, and the filled circles photographic data from the Palomar Schmidt telescope.

Our CCD photometric observations of Parsamian 21 in the period 2003 - 2017 show that the brightness of the star is almost steady. We observed only low amplitude fluctuations around the middle values. Comparing our 
CCD photometric observations with the data from Palomar plates showed no significant change in the brightness of the star for a very long period (65 years). We conclude that Parsamian 21 is probably a member of the group of long-lived FUors and that the time-scale of the FUor phenomenon in some cases is much longer than that predicted in previous studies.

\section{Summary}

Photometric results presented in this paper show the usefulness of systematical photometric monitoring of the regions of star formation. These data can be used to detect new FUor or EXor events and to determine the type of the registered outbursts. Therefore, the collection of new photometric data (from photographic plate archives and ongoing photometric monitoring) will be of great importance for study of PMS variability. At the same time, according to existing observations the light curves of FUor objects, confirming the hypothesis that each known FUor has a different rate of increase and decrease in brightness and a different light curve shape.

\section{Acknowledgements}

The authors thank the Director of Skinakas Observatory Prof. I. Papamastorakis and Prof. I. Papadakis for the award of telescope time. This work was partly supported by the Bulgarian Scientific Research Fund of the Ministry of Education and Science under the grants DM 08-2/2016, DN 08-1/2016, DN 08-20/2016 and DN 18-13/2017 as well as by the project RD-08-112/2018 of the University of Shumen. This research has made use of the NASA's Astrophysics Data System Abstract Service, the SIMBAD database and the VizieR catalogue access tool, operated at CDS, Strasbourg, France. Some of the data presented in this paper were obtained from the Mikulski Archive for Space Telescopes (MAST). STScI is operated by the Association of Universities for Research in Astronomy, Inc., under NASA contract NAS5-26555.

\section{References}

Ambartsumyan, V. A. 1971, Astrophysics, 7, 331

Aspin, C.; Reipurth, B. 2009, AJ, 138, 1137

Aspin, C.; Reipurth, B.; Beck, T. L. et al. 2009, ApJ, 692, L67

Audard, M., Ábrahám, P., Dunham, M. M., et al. 2014, in Protostars and Planets VI, ed. H.

Beuther et al. (Tucson, AZ: Univ. Arizona Press), p. 387

Briceño, C., Vivas, A. K., Hernandez, J. et al. 2004, ApJ, 606, L123

Clarke, C.; Lodato, G.; Melnikov, S. Y.; Ibrahimov, M. A. 2005, MNRAS, 361, 942

Connelley, M. S.; Reipurth, B. 2018, ApJ, 861, 145

Elias, J. H. 1978, ApJ, 223, 859

Greene, T. P.; Aspin, C.; Reipurth, B. 2008, AJ, 135, 1421

Hartmann, L.; Kenyon, S. J. 1996, ARA\&A, 34, 207

Herbig, G. H. 1977, ApJ, 217, 693

Herbig, G. H. 2008, AJ, 135, 637

Herbst, W.; Herbst, D. K.; Grossman, E. J.; Weinstein, D. 1994, ApJ, 108, 1906

Ibryamov, S. I.; Semkov, E. H.; Peneva, S. P. 2015, PASA, 32, id. e021

Kóspál, Á.; Ábrahám, P.; Apai, D. et al. 2008, MNRAS, 383, 1015

Kóspál, Á.; Ábrahám, P.; Acosta-Pulido, J. A. et al., 2016, A\&A, 596, A52

Kun, M. 2008, IBVS, 5850, 1

Lee, J.-E., Kang, W., Lee, S.-G. et al. 2011, JKAS, 44, 39

McNeil, J. W. 2004, IAU Circ., 8284 
Mickaelian, A. M.; Gigoyan, K. S.; Gyulzadyan, M. V. et al. 2017, ComBAO, 64, 102

Miller, A, A.; Hillenbrand, L. A.; Covey, K. R. et al., 2011, ApJ, 730, 80

Munari, U.; Siviero, A.; Ochner, P.; Fiorucci, M.; Dallaporta, S. 2009, CBET, 1898, 1

Parsamian E. S. 1965, Izv. Akad. Nauk Armyan. SSR., Ser. Fiz.-Math., 18, 146

Peneva, S. P.; Semkov, E. H.; Munari, U.; Birkle, K. 2010, A\&A, 515, A24

Peneva, S. P.; Semkov, E. H.; Stavrev, K. Y. 2009, Ap\&SS, 323, 329

Reipurth, B.; Hartmann, L.; Kenyon, S. J.; Smette, A.; Bouchet, P. 2002, AJ, 124, 2194

Reipurth, B.; Aspin, C., 2010, in Evolution of Cosmic Objects through their Physical Activity, eds. H. A. Harutyunian, A. M. Mickaelian, Y. Terzian (Yerevan: Gitutyun), p. 19

Reipurth, B.; Aspin, C.; Beck, T. et al. 2007, AJ, 133, 1000

Samus, N. 2009, CBET, 1896, 1

Semkov, E. H.; Peneva, S. P.; Munari, U.; Milani, A.; Valisa, P. 2010, A\&A, 523, L3

Semkov, E. H.; Peneva, S. P.; Munari, U. et al. 2012, A\&A, 542, A43

Semkov, E. H.; Peneva, S. P.; Munari, U. et al. 2013, A\&A, 556, A60

Semkov, E. H.; Peneva, S. P.; Ibryamov, S. I. 2017, Bulg. Ast. J., 26, 57

Staude, H. J.; Neckel, Th., 1992, ApJ, 400, 556 